
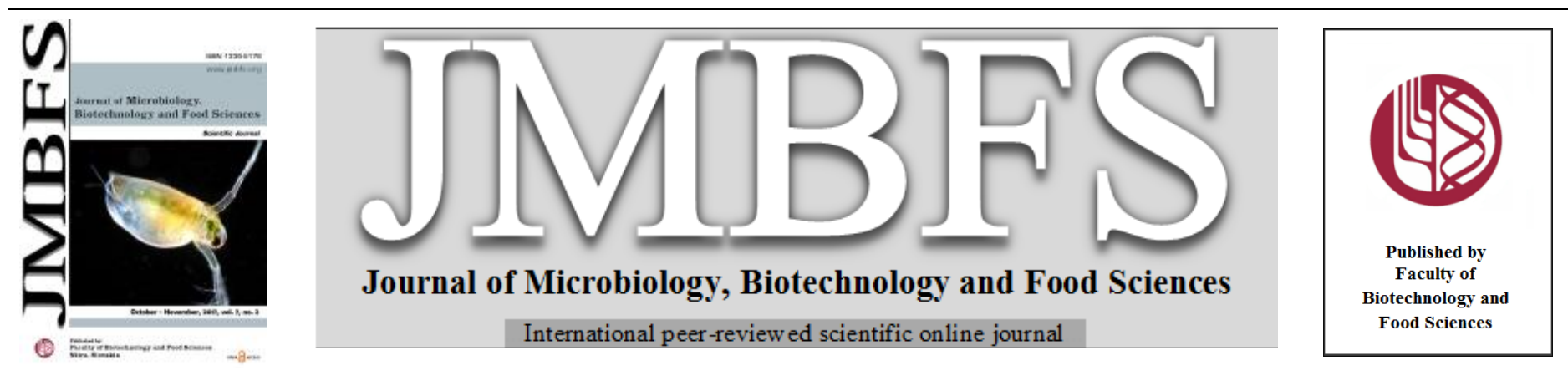

\title{
ETHANOL PRODUCTION AND SUGAR CONSUMPTION OF CO-CULTURE Saccharomyces cerevisiae FNCC 3012 WITH Candida tropicalis FNCC 3033 IN MEDIA CONTAINING INHIBITOR FERMENTATION
}

\section{Tatang Sopandi ${ }^{1}$, A. Wardah ${ }^{2}$}

Address(es):

${ }^{1}$ Department of Biology, Faculty of Mathematical and Natural Science, University of PGRI Adi Buana, Surabaya, Indonesia.

Jl, Dukuh Menanggal XII, 60234, Surabaya, East Java, Indonesia.

${ }^{2}$ Faculty of Economy, University of 17 Agustus 1945, Surabaya, Indonesia. J1, Semolowaru 45, 60119, Surabaya, East Java, Indonesia.

*Corresponding author: tatang_sopandi@yahoo.co.id

doi: 10.15414/jmbfs.2017.7.2.160-167

\section{ARTICLE INFO}

Received 12.1.2016

Revised 12. 7. 2017

Accepted 12. 9. 2017

Published 1. 10. 2017

Regular article OPEN $\partial$ ACCESS

\begin{abstract}
Inhibitor fermentation is one of the problems that arise in the ethanol production from lignocellulose waste. This work examined the ethanol yield and sugar consumption of mono and co-culture S. cerevisiae with C. tropicalis in media containing inhibitor fermentation. Furfural and phenol were used for inhibitor fermentation in basal medium with concentrations of 2.0 and $5.0 \%$, respectively. The basal

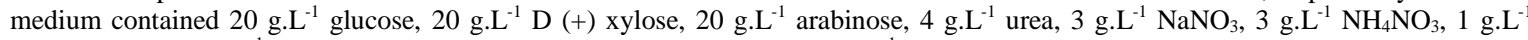
$\mathrm{KH}_{3} \mathrm{PO}_{4}$ and $0.7 \mathrm{~g} . \mathrm{L}^{-1} \mathrm{MgSO}_{4} \cdot 7 \mathrm{H}_{2} \mathrm{O}$ with $\mathrm{pH}$ adjusted to 5.5 with $1 \mathrm{~mol} . \mathrm{L}^{-1} \mathrm{HCl}$. After furfural or phenol addition separately, and inoculation by mono and co-culture $S$. cerevisiae FNCC 3012 with C. tropicalis FNCC 3033, all media were incubated at $28-29{ }^{\circ} \mathrm{C}$, $50 \% \mathrm{r}$.h. in the dark for 5 days in a rotary incubator at $60 \mathrm{rev} / \mathrm{min}$. We found yeast colony count, sugar consumption, ethanol yields and efficiency of fermentation by co-culture S. cerevisiae with $C$. tropicalis higher than mono-culture S. cerevisiae or C.tropicalis in the fermentation media with or without inhibitors. This work indicated that co-culture $S$. cerevisiae with $C$. tropicalis were more tolerant to furfural and phenol. Ethanol yield $8.52 \%, 5.37 \%$ and $3.83 \%$ obtained from basal medium, basal medium plus 2.5 or $5.0 \%$ furfural, respectively and efficiency of fermentation $27.00 \%, 17.00 \%$ and $12.20 \%$. Ethanol yields $8.13 \%, 5.62 \%$ and $3.19 \%$ obtained from basal medium, basal medium plus 2.5 or $5.0 \%$ phenol, respectvely and efficiency of fermentation $28.20 \%, 20.00 \%$ and $14.00 \%$. Coculture $S$. cerevisiae FNCC 3012 with C. tropicalis 3033 demonstrated potential as a fermentation process for ethanol production from lignocellulosic media content inhibitors. The use of this co-culture effectively utilizes hexose and pentose sugars in the substrate, increasing the yield and efficiency of fermentation for ethanol production.
\end{abstract}

Keywords: co-culture, fermentation, ethanol, inhibitor, S.cerevisiae and C.tropicalis

\section{INTRODUCTION}

The limitations of fossil fuels and environmental conditions have drawn attention to development of alternative energy sources that have a lower impact on the environment (Abreu-Cavalheiro and Monteiro, 2013). Ethanol is an alternative energy source with the potential to replace fossil energy sources and has received a lot of attention over the past few years (Chen, 2011). Ethanol can be produced from various agricultural raw materials including lignocellulose (Balat, 2011; Tesfaw and Assefa, 2014). Due to its renewability, large quantities, relatively low prices compared to grain or sugar and potentia environmental benefits, lignocellulosic biomass has been considered a possible raw material for ethanol production (Cardona and Sánchez, 2007; Kumar et al., 2008; Lee and Huang, 2000; Mielenz, 2001; Service, 2007; Zaldivar et al., 2001). Lignocellulosic biomass is preferred as a raw material for ethanol production over sugar or starch derived from crop products because it does not compete in terms of food needs and concerns the utilization of agricultural residue (Gutiérrez-Rivera et al., 2012; Ishola et al., 2014).

Efficient fermentation of ethanol production from lignocellulosic biomass is affected by the consumption of glucose and xylose, which are the main products of lignocellulosic hydrolysates (Lee and Huang, 2000; Service, 2007; Eiteman et al., 2008). However, the lack of a microorganism capable of efficiently fermenting all sugars released by hydrolysis from lignocellulosic materials has been one of the main factors preventing the utilization of lignocellulose (Zaldivar et al., 2001). Saccharomyces cerevisiae is the dominant yeast used for ethanol production but cannot metabolize xylose and convert it into ethanol (Jeffries and Jin, 2004; Lin and Tanakan, 2006).

In addition, another problem associated with efficient conversion of cellulose and hemicellulose sugars to ethanol is that during dilute acid hydrolysis a broad range of compounds that inhibit the fermenting microorganism are liberated or formed along with the sugars (Larsson et al., 2001). The presence of inhibiting compounds, such as weak acids, furans and phenolic compounds, that are formed or released during thermochemical pretreatment steps such as acid and steam explosion can decrease the ethanol yield and productivity of lignocellulosic fermentation (Parawira and Tekere, 2011). Reduction of the ethanol yield and productivity by inhibiting components can influence the performance of microorganisms during the fermentation stage (Almeida et al., 2007).

The choice of the fermenting microorganism, complete substrate utilization, inhibitor tolerance and ethanol productivity are important aspects in the production of ethanol from lignocellulose (Bettiga et al., 2009). Microorganisms that consume sugars such as glucose and xylose sequentially must have lower productivities for the generation of a product than if the organism were to consume the sugars simultaneously (Zaldivar et al., 2001). For economical bioethanol production from lignocellulosic materials, the microorganism should use all glucose and xylose in the lignocellulose hydrolysate efficiently and it should have high tolerance to the inhibitors present in the lignocellulose hydrolysate (Cheng et al., 2014).

Strategies for using a single microorganism to convert glucose and xylose simultaneously have limitations (Eiteman et al., 2008). Co-culture among microorganisms could potentially increase ethanol production and the efficiency of fermentation from lignocellulosic hydrolysate. Co-culture of $S$. cerevisiae and other microorganisms reduced inhibitory compounds in lignocellulosic hydrolysates (Taherzadeh et al., 2013; Wan et al., 2012), increased ethanol yield and production rate (Singh et al., 2014; Wan 2012), shortens fermentation time and reduced process cost (Hickert et al., 2013; Tesfaw and Assefa, 2014) Co-culture of S.cerevisiae with $C$. tropicalis has the ability to generate and convert fermentable sugars from a waste stream rice husk to ethanol (Sopandi and Wardah, 2015). This work examined the ethanol yield and sugar consumption of mono and co-culture $S$. cerevisiae with $C$. tropicalis in medium containing inhibitor fermentation. 


\section{MATERIAL AND METHODS}

\section{Culture of microorganism}

Saccharomyces cerevisiae FNCC 3012 and Candida tropicalis FNCC 3033 were obtained from Microbiology Laboratories, PPAU Gadjah Mada University, Yogyakarta, Indonesia. Saubroad agar (Oxoid) was used to maintain the strains $S$. cerevisiae and C. tropicalis. Working stock cultures were prepared from stock for 7 days at $28{ }^{\circ} \mathrm{C}$ in SA plate subcultures from the master stock. Colonies were aseptically sampled by scraping the top with an inoculating loop and transferring to $10 \mathrm{ml}$ sterile water. Inoculum stock suspension was prepared from working stock and diluted to $1.7 \times 10^{6}$ cell. $\mathrm{mL}^{-1}$, as enumerated, using a haemocytometer.

\section{Fermentation}

The batch fermentation experiments were carried out in a $250 \mathrm{~mL}$ Erlenmeyer flask with working volumes of $100 \mathrm{~mL}$. The basal medium contained $20.0 \mathrm{~g} . \mathrm{L}^{-1}$ glucose, 20.0 g.L $\mathrm{L}^{-1} \mathrm{D}(+)$ xylose, 20.0 g.L $\mathrm{L}^{-1}$ arabinose, 4.0 g.L $\mathrm{L}^{-1}$ urea, 3.0 g.L $\mathrm{L}^{-1}$ $\mathrm{NaNO}_{3}, 3.0$ g.L. $\mathrm{NH}_{4} \mathrm{NO}_{3}, 1.0$ g.L $\mathrm{L}^{-1} \mathrm{KH}_{3} \mathrm{PO}_{4}$ and 0.7 g.L $\mathrm{LgSO}_{4} \cdot 7 \mathrm{H}_{2} \mathrm{O}$ with pH adjusted to 5.5 with 1.0 mol. $\mathrm{L}^{-1} \mathrm{HCl}$. Liquid basal medium $(9.0$ 1) was mixed thoroughly and $100 \mathrm{ml}$ individually dispensed into $250 \mathrm{~mL}$ Erlenmeyer flasks, autoclaved and cooled to room temperature. Media in Erlenmeyer were divided into two groups, Individually flask, one group was added furfural until final concentrations of $0.0,2.5$ and $5.0 \%$ and another group was added phenol unti final concentration of $0.0,2.5$, and $5.0 \%$. A $1.0 \mathrm{~mL}$ inoculum stock suspension of $S$. cerevisiae and $1.0 \mathrm{~mL}$ C. tropicalis for mono-culture were aseptically dispensed into individual Erlenmeyer flasksand $0.5 \mathrm{~mL} S$. cerevisiae with $0.5 \mathrm{~mL}$ C. tropicalis for co-culture were added into the flasks and incubated at $28-29{ }^{\circ} \mathrm{C}$ $50 \%$ r.h. in the dark for $5 \mathrm{~d}$ in a rotary incubator at $60 \mathrm{rev} / \mathrm{min}$. This inoculation and incubation method was used for all cultivation in this study.

\section{Yeast count}

Serial dilution $10^{10}$ using sterile water was conducted to yeast count observation in $10 \mathrm{~mL}$ media before and after 5 days fermentation. Each serial dilution $(0.1$ $\mathrm{mL}$ ) was inoculated and spread onto Saubroad agar (Oxoid), and incubated at 28 $29{ }^{\circ} \mathrm{C}, 50 \% \mathrm{r}$.h. in the dark for $3 \mathrm{~d}$. A colony counter was used for counting colonies on the media.

\section{Determination of ethanol}

Ethanol was measured using a gas chromatograph Carbomax t70-10-0 column, an FID t220 detector, with helium as carrier gas with a flow rate of $40.3 \mathrm{~mL}$.min ${ }^{1}$, and a tin column Porapack $\mathrm{Q}$, with a detector temperature of $160{ }^{\circ} \mathrm{C}$ and a column temperature of $180{ }^{\circ} \mathrm{C}$ with an injection volume of $1.0 \mu \mathrm{L}$. Fermented media were filtered through Whatman Grade 1 paper.

\section{Determination of sugar}

Glucose, $\mathrm{D}(+)$ xylose and arabinose were determined using HPLC (Shimadzu, Kyoto, Japan) at $85{ }^{\circ} \mathrm{C}$, a Metacharb $87 \mathrm{C}$ column, with $\mathrm{H}_{2} \mathrm{O}$ as eluent, with a flow rate of $0.6 \mathrm{~mL} / \mathrm{min}$ and an RID detector. After fermentation, the media were mixed and aseptically filtered through Whatman Grade 1 paper. The filtrate was centrifuged at $12,000 \mathrm{rpm}$ for $15 \mathrm{~min}$, refiltered through millex $0.45 \mu \mathrm{m}$ and 25 $\mu \mathrm{L}$ of sample was injected for HPLC. Glucose, $\mathrm{D}(+)$ xylose and arabinose (Merck) were used as standard with concentrations of 62.5, 125, 250 and 500 ppm, respectively.

\section{Efficiency of fermentation}

To determine the efficiency of the fermentation of ethanol production by mono and co-culture of S. cerevisiae and C. tropicalis, we used the following formula:

EFbiomass $(\%)=\frac{\text { Ethanol yield }(L)}{\text { Amount sugar before fermentation }(g) * 0,511} \times 100$

\section{Statistical analysis}

Tukey's honestly significant difference multiple comparison test and a paired sample t-test were used to segregate significantly different treatment using SPSS 16 software. Analysis of variance (ANOVA) was performed to determine differences between experiments with $5 \%$ level of significance $(\mathrm{P}<0.05)$.

\section{RESULTS AND DISCUSSION}

\section{Yeast count}

Addition of furfural (Fig. 1A) to the growth medium significantly ( $\mathrm{p}<0.05$ ) decreased the yeast colony count in mono- or co-culture $S$. cerevisiae with $C$. tropicalis. This work indicated that furfural inhibits the growth of yeast in monoculture and co-culture S.cerevisiae with $C$. tropicalis. Some investigators have reported the effect of furfural on the growth of microorganisms. Palmqvist and Hahn-Hägerdal (2000) reported that furfural inhibited the specific growth and fermentation rate of yeasts. Agbogbo et al. (2007) reported that a concentration of furfural of $1.5 \mathrm{~g} . \mathrm{L}^{-1}$ could interfere with the respiration and growth of microorganisms. Hristozova et al. (2000) reported that a concentration of furfural of $0.04 \%$ inhibited glutamate dehydrogenase and ion ammonia assimilation in the alanine metabolism of C.blankii 35 and C. pseudotropicalis 11. Kelly et al. (2008) reported that a concentration of furfural of 1 g.L $\mathrm{L}^{-1}$ or higher inhibited growth of C. guilliermondii. Jones (1989) and Almeida et al. (2009) suggested that furfural and 5-hydroxymethyl furfural (HMF) can consumed by $S$. cerevisiae but will lose ATP. Mattam et al. (2016) reported that an increase in the level of furfural, HMF and acetic acid in growth media led to a gradual decrease in $C$. tropicalis biomass.
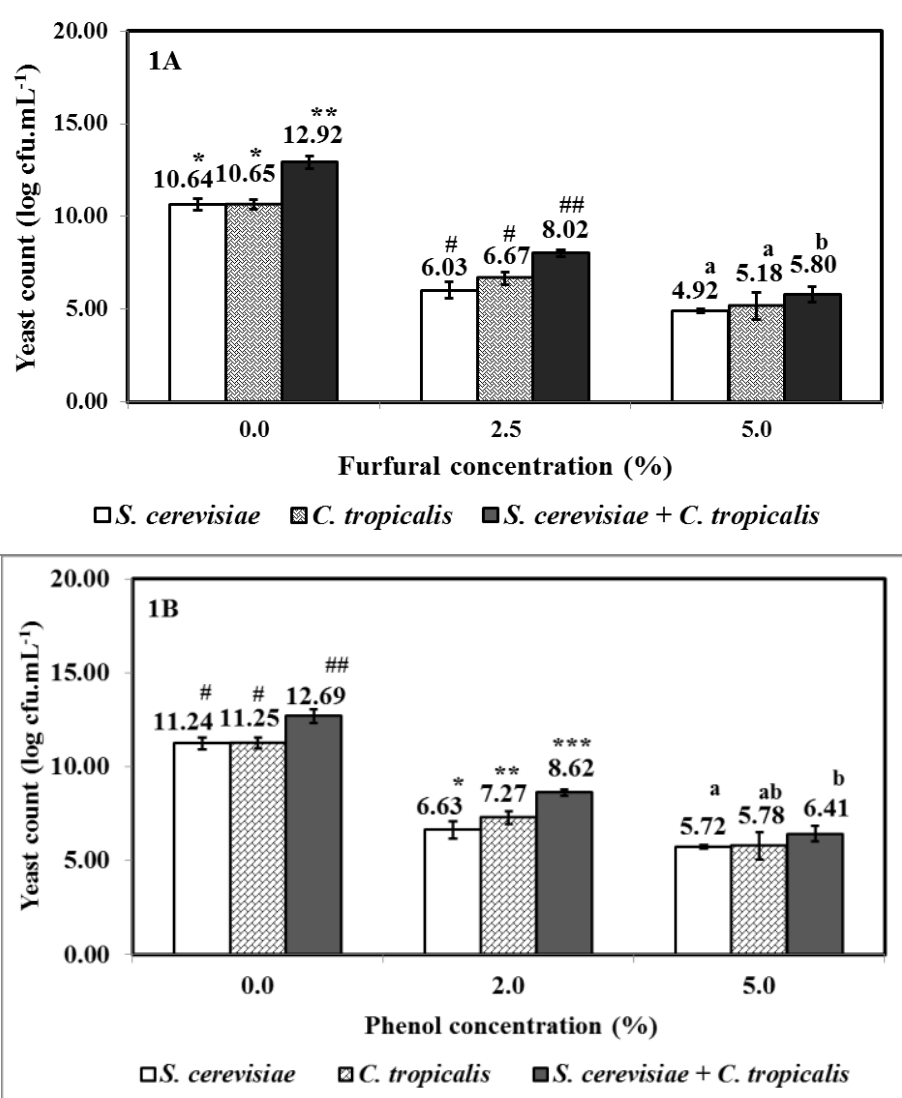

Figure 1 Yeast count in medium plus different concentrations of furfural $(1 \mathrm{~A})$ and phenol (1B) after 5 days' fermentation by mono and co-culture $S$. cerevisiae with $C$. tropicalis. The values with different superscripts ${ }^{\mathrm{a}}{ }^{\mathrm{and}}{ }^{\mathrm{b}}$, * and $* *$ or ${ }^{\#}$ and ${ }^{\# \#}$ ) are different significant $(p<0.05)$ of means from five independent observations in the same furfural concentration.

However, this work shows that the yeast count colony in co-culture of $S$ cerevisiea with $C$. tropicalis is significantly $(\mathrm{P}<0.05)$ higher than mono-culture $S$. cerevisiae or $C$. tropicalis in the medium growth with or without furfural. We hypothesized that there is a synergistic mechanism to stimulate yeast growth through simultaneous utilization of fermentable sugars such as glucose and xylose and degradation of furfural in growth medium by co-culture of $S$. cerevisiae with $C$. tropicalis. Some investigators have reported consumption of fermentable sugars (glucose and xylose) simultaneously by co-culture fermentation. Hikert et al. (2013) reported that co-culture of $C$. shehatae HM 52.2 with $S$. cerevisiae ICV D254 can simultaneously consume glucose and xylose in synthetic medium and rice hull hydrolysate. Fu and Peiris (2008) reported that co-culture of Zymomonas mobilis with Pachysolen tannophilus was fully consumed in a mixture of glucose and xylose media. Some investigators have reported the degradation and conversion of furfural by yeast. Taherzadeh et al. (1999) reported that $S$. cerevisiae converted furfural to furfuryl alcohol in exponentially growing cells. Cheng et al. (2014) reported that $C$. tropicalis W103 degraded furfural and 5-hydromethylfurfural under aerobic conditions after $60 \mathrm{~h}$ of aerobic incubation. Some investigators reported that coculture of $S$. cerevisiae with other microbes can reduce inhibitory compounds in lignocellulosic hydrolysates. Tomás et al. (2013) reported that co-culture of Thermoanaerobacter pentosaceus with $S$. cerevisiae was able to gro and metabolize furfural up to $0.5 \mathrm{~g} . \mathrm{L}^{-1}$ in the liquid fraction of alkaline-peroxidepretreated rapeseed straw. Wan et al. (2012) reported that co-cultures of $S$ cerevisiae Y5 with Pichia stipitis CBS6054 can grow and metabolize furfural and $\mathrm{HMF}$ in a medium of a mixture of xylose and glucose. 
Addition of phenol (Fig. 1B) to the growth medium significantly ( $\mathrm{p}<0.05$ ) decreased the yeast colony count in mono- or co-culture $S$. cerevisiae with $C$. tropicalis. This work indicated that phenol inhibits the growth of yeast in monoculture and co-culture $S$. cerevisiae with $C$. tropicalis. Some investigators have reported the inhibitory effect of phenol on the growth of microorganisms. Heipieper et al. (1994) reported that phenol can degrade cell membrane integrity and decrease membrane affection as a selective buffer. Ding et al. (2011) sugested that acetic acid, furfural and phenol are main inhibitors of growth, fermentation and some yeast metabolites. Some fermentation inhibitors such as HMF and phenol can inhibit yeast metabolism (Almeida et al., 2009; Larsson et al., 1999; Palmqvist and Hahn-Hägerdal, 2000; Sluiter et al., 2010). However, Paca et al. (2002) suggested that $C$. tropicalis can use phenol as a source of carbon and energy. Adeboye et al. (2014) reported that phenolic compounds can exhibit lag phase elongation and a decreased maximum specific growth rate of $S$. cerevisiae. Pizzolitto et al. (2015) reported an inhibitory effect of phenol on the growth parameters of Aspergillus parasiticus depending on the compound assayed and its concentration in the medium. Similarly to furfural, this work showed a yeast count colony in co-culture of S.cerevisiae with $C$. tropicalis significantly $(\mathrm{P}<0.05)$ higher than mono-culture of S.cerevisiae or $C$. tropicalis in the medium with or without phenol. We hypothesized that there is a synergistic mechanism to stimulate yeast growth through simultaneous utilization of fermentable sugars and degradation of phenol in the growth medium by coculture of S. cerevisiae with C. tropicalis. Some investigators have reported that C. tropicalis can degrade the phenol component (Ahuatzi-Chacon et al., 2004; Komarkova et al., 2003; Krug et al., 1985; Wang et al., 2012). Jönsson et al. (2013) and Larsson et al. (2000) reported that $S$. cerevisiae can convert some inhibitory phenolics to less toxic compounds such as coniferyl aldehyde through convertion to coniferyl alcohol and dihydroconiferyl alcohol. Kuntiya et al. (2013) reported that phenol can be degraded and used as a source of carbon energy by $C$. tropicalis.

\section{Residue and sugar consumption}

Addition of furfural to the growth medium significantly $(\mathrm{p}<0.05)$ influenced glucose residue (Fig. 3) and xylose (Fig. 4), but did not significantly ( $p>0.05$ ) influence arabinose residue (Fig. 5A). Addition of furfural to the growth medium also significantly $(\mathrm{p}<0.05)$ decreased glucose (Fig. 3B) and xylose (Fig. 4B) consumption, but did not significantly $(\mathrm{p}>0.05)$ influence arabinose consumption (Fig. 5B). Glucose, fructose and mannose are fermented via the Embden-Meyerhof pathway of glycolysis, and galactose requires the Leloir pathway (Wendland et al., 2009). Effects of inhibitory furfural and phenol on glucose consumption were reported by several researchers (Almeida et al., 2009: Larsson et al., 1999; Lin et al., 2015; Palmqvist et al., 2000; Sluiter et al., 2010). Wikandari et al. (2010) reported that glucose consumed by $S$. cerevisiae isolate of Bekonang only 34.94 and $1.93 \%$ in the medium containing 1.0 and $1.5 \mathrm{~g} . \mathrm{L}^{-1}$ of furfural, respectively.

However, this work showed that glucose consumption by co-culture of $S$. cerevisiae with $C$. tropicalis was significantly $(\mathrm{P}<0.05)$ higher than monoculture $S$. cerevisiae or $C$. tropicalis in the medium with or without furfural. It was suspected that the higher glucose consumption by co-culture than monoculture in this study was due to degradation of furfural by each yeast in the mixture fermentation. Under anaerobic conditions, $S$. cerevisiae can convert furfural to furfuryl alcohol (Diaz de Villegas et al., 1992; Sárvári Horváth et al., 2003) and the reduction of furfural has been linked to the co-factor NADH (Wahlbom et al., 2002)

In addition, the higher sugar consumption by co-culture than mono-culture in this study was also suspected to be due to the contribution of glucose consumption by C. tropicalis in the substrate mixture of glucose and xylose. In this work, $C$. tropicalis can consume glucose from media, although less than S. cerevisiae. This observation similar to that of Panchal et al. (1988) and du Preez et al. (1986), who reported a diauxic (sequential) consumption of D-glucose and D-xylose in the same order by $C$. shehatae and $P$. stipites when using mixtures of these sugars in the culture medium. Laplace et al. (1993) reported that co-culture of $C$. shehatae with $S$. cerevisiae completely consumed D-glucose from a mixture medium containing $70 \%$ of D-glucose and $30 \%$ of D-xylose after $14 \mathrm{~h}$ fermentation, while D-xylose, in practice, was not consumed. These researchers suggest that xylose consumption by $C$. shehatae can be inhibited in the presence of glucose.

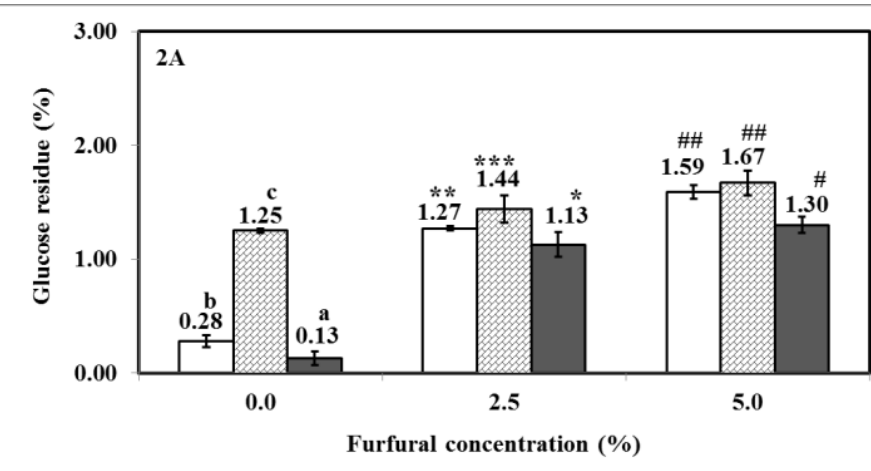

$\square$ S. cerevisiae $\quad \square$ C. tropicalis $\quad \square$ S. cerevisiae + C. tropicalis

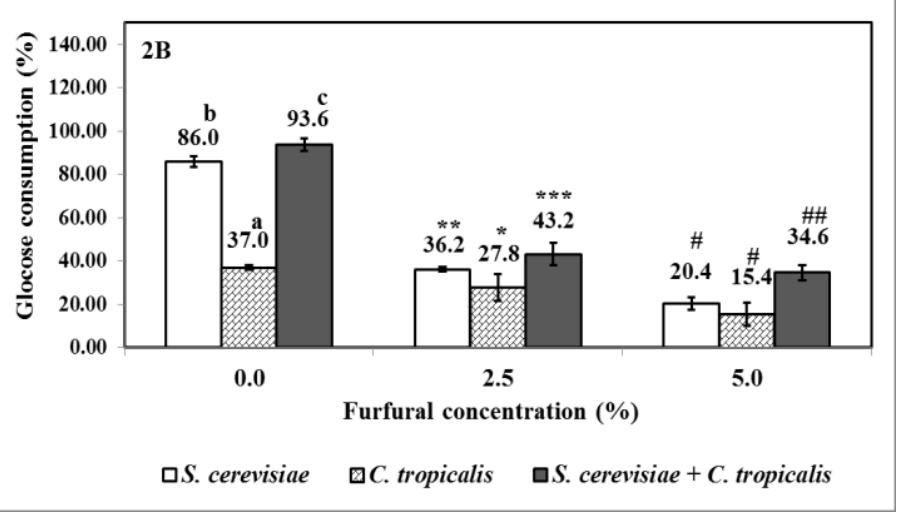

Figure 2 Glucose residue (2A) and consumption of glucose (2B) after 5 days fermentation by mono- and co-culture of $S$. cerevisiae with $C$. tropicalis in the media plus different furfural concentrations. The values with different

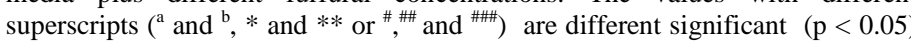
of means from five independent observations in the same furfural concentration.
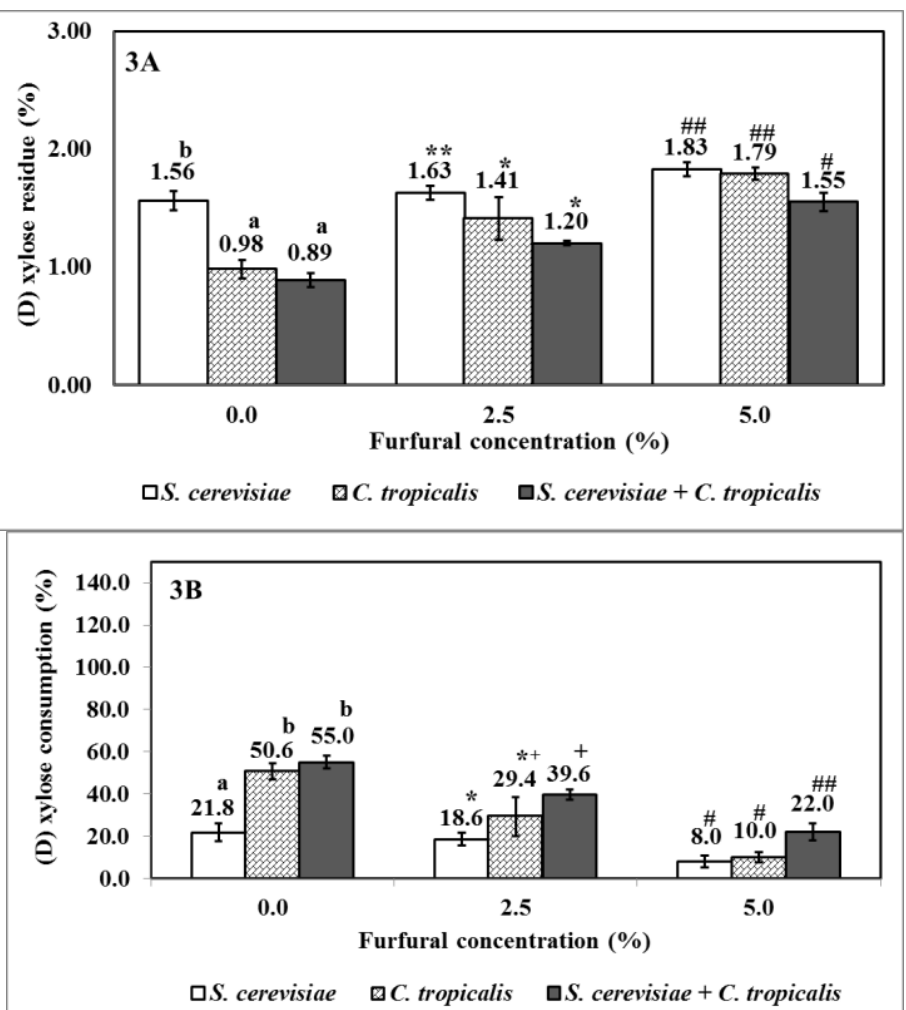

Figure 3 (D) xylose residue (3A) and consumption of xylose (3B) after 5 days fermentation by mono- and co-culture of $S$. cerevisiae with $C$. tropicalis in media plus different furfural concentrations. The values with different superscripts ${ }^{\mathrm{a}}$ and $^{\mathrm{b}}, *$ and $* *$ or ${ }^{\#},{ }^{\#}$ and $\left.{ }^{\# \#}\right)$ are different significant $(\mathrm{p}<0.05)$ of means from five independent observations in the same furfural concentration 

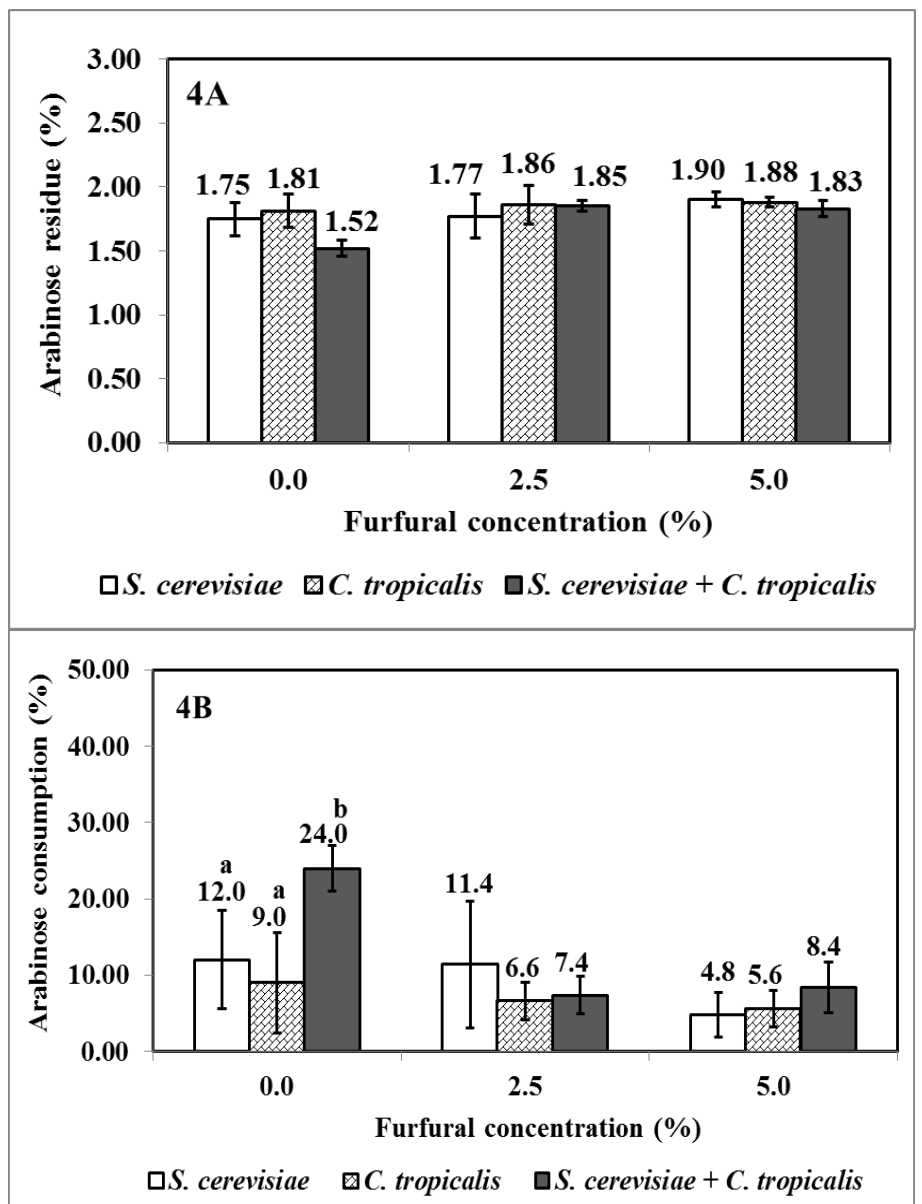

Figure 4 Arabinose residue (4A) and consumption of arabinose (4B) after 5 days' fermentation by mono- and co-culture of $S$. cerevisiae with $C$. tropicalis in media plus different furfural concentrations. The values with different superscripts $\left({ }^{a}\right.$ and $\left.{ }^{b}\right)$ are different significant $(p<0.05)$ of means from five independent observations in the same furfural concentration

In this work, $\mathrm{D}(+)$ xylose consumption by mono-culture of $C$. tropicalis and coculture of $S$. cerevisiae with $C$. tropicalis was higher than $C$. tropicalis. Cheng et al. (2014) reported that Candida tropicalis W103 was able to use xylose as the carbon source for cell growth under aerobic or anaerobic conditions, and when glucose was used as the carbon source, ethanol was produced under aerobic or anaerobic conditions, but $C$. tropicalis grew slightly slower under anaerobic conditions than under aerobic conditions and displayed sequential sugar consumption, first utilizing glucose and then xylose. Higher $\mathrm{D}(+)$ xylose consumption by co-culture than mono-culture in this study was allegedly due to inhibitor degradation by each yeast in the mixture fermentation as described before, as well as the contribution of $S$. cerevisiae in consuming $\mathrm{D}(+)$ xylose Native $S$. cerevisiae does not metabolize xylose (Jeffries and Jin, 2004; Lin and Tanakan, 2006) and nearly all reported xylose isomerase-based pathways in $S$ Cerevisiae suffer from poor ethanol productivity, low xylose consumption rates and poor cell growth compared with an oxidoreductase pathway and, additionally, often require adaptive strain evolution (Lee et al., 2012). As all yeasts of the genus Saccharomyces lack the gene that produces the enzyme xylose isomerase (Van Maris et al., 2006), conversion of xylose to xylulose is necessary for carbon uptake (Chiang et al., 1981; Gong et al., 1981). Although low $(21.80 \%)$, this work indicates that $S$. cerevisiae can consume $D(+)$ xylose, allegedly due to the lack of glucose in culture medium, as a mechanism of adaptation to nutritional deficiencies or our $S$. cerevisiae has undergone mutations in fermentation conditions. Figure 5 shows the glucose, $\mathrm{D}(+)$ xylose and arabinose residues in the media after being fermented by $S$. cerevisiae. Shin et al. (2015) suggested that $S$. cerevisiae is able to ferment xylose but first utilizes D-glucose before the D-xylose can be transported and metabolized.

Addition of furfural to the growth medium significantly ( $p<0.05$ ) decreased arabinose consumption by mono- or co-culture $S$. cerevisiae with $C$. tropicalis in the fermentation media. Co-culture of $S$. cerevisiae with $C$. tropicalis significantly $(\mathrm{p}<0.05)$ increased arabinose consumption in the fermentation media. Schimer-Michel et al. (2008) argued that arabinose was metabolized in a later phase, when both glucose and xylose were exhausted. Generally, in this work $S$. cerevisiae and $C$. tropicalis shown very low consumption of arabinose in the media with or without furfural. We found that arabinose consumption depends on the availability of glucose and xylose in the media.

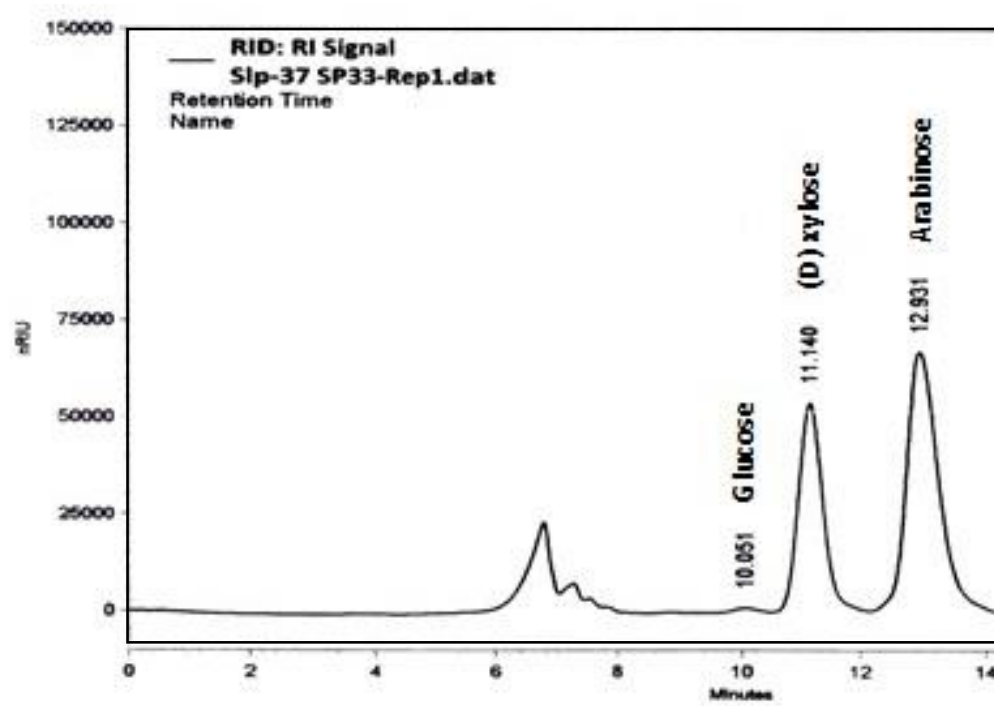

Figure 5. An HPLC chromatogram of residue glucose, D(+) xylose and arabinose in medium after 5 days' fermentation by $\quad$ S. cerevisiae.

Addition of phenol to the growth medium significantly $(\mathrm{p}<0.05)$ influnced glucose (Fig. 6A) and xylose (Fig. 7A) residue, but did not significantly ( $\mathrm{p}>$ 0.05 ) influence arabinose residue (Fig. 8A). Addition of phenol to the growth medium also significantly ( $<<0.05$ ) decreased glucose (Fig. 6B) and xylose (Fig. 7B) consumption, but did not significantly $(\mathrm{p}>0.05)$ affect arabinose consumption (Fig. 8B). The toxicity of phenolics is very variable as it depends on the functional groups (Adeboye et al., 2014; Ando et al., 1986; Jonsson et al. 2013); more methoxy groups are related to high hydrophobicity and toxicity (Klinke et al., 2004). Yeast S. cerevisiae can assimilate many phenolics which can be part of the detoxification process occurring during fermentation (Delgenes et al., 1996; Mills et al., 1971). Phenolic compounds mainly interfere with the function of proteins and trigger changes in the protein-to-lipid ratio (Keweloh et al., 1990). Hence, these compounds affect cellular functions like sorting and signalling, and also cause membrane swelling (Caspeta et al., 2015).

Richard et al. (2003) stated that the fungal pathways L-arabinose and D-xylose to convert L-arabinose and D-xylose to D-xylulose 5-phosphate go through oxidation and reduction reactions before they are phosphorylated by xylulokinase. D-xylose is first reduced to xylitol by a reduced nicotinamide adenine dinucleotide phosphate (NADPH)-consuming reaction. Xylitol will be oxidized by an NADb-consuming reaction to form $\mathrm{D}$-xylulose. In fungi, $\mathrm{L}$ arabinose goes through four redox reactions. Two oxidations are coupled to NADp consumption and two reductions to NADPH consumption. Futhermore, Richard et al. (2003) reported that $S$. cerevisiae enables growth on L-arabinose and under anaerobic conditions ethanol is produced from L-arabinose, but at a very low rate. Similarly to furfural, in this work $S$. cerevisiae and $C$. tropicalis shown very low consumption of arabinose in the media with or without phenol. We found that arabinose consumption depends on the availability of glucose and xylose in the media. 

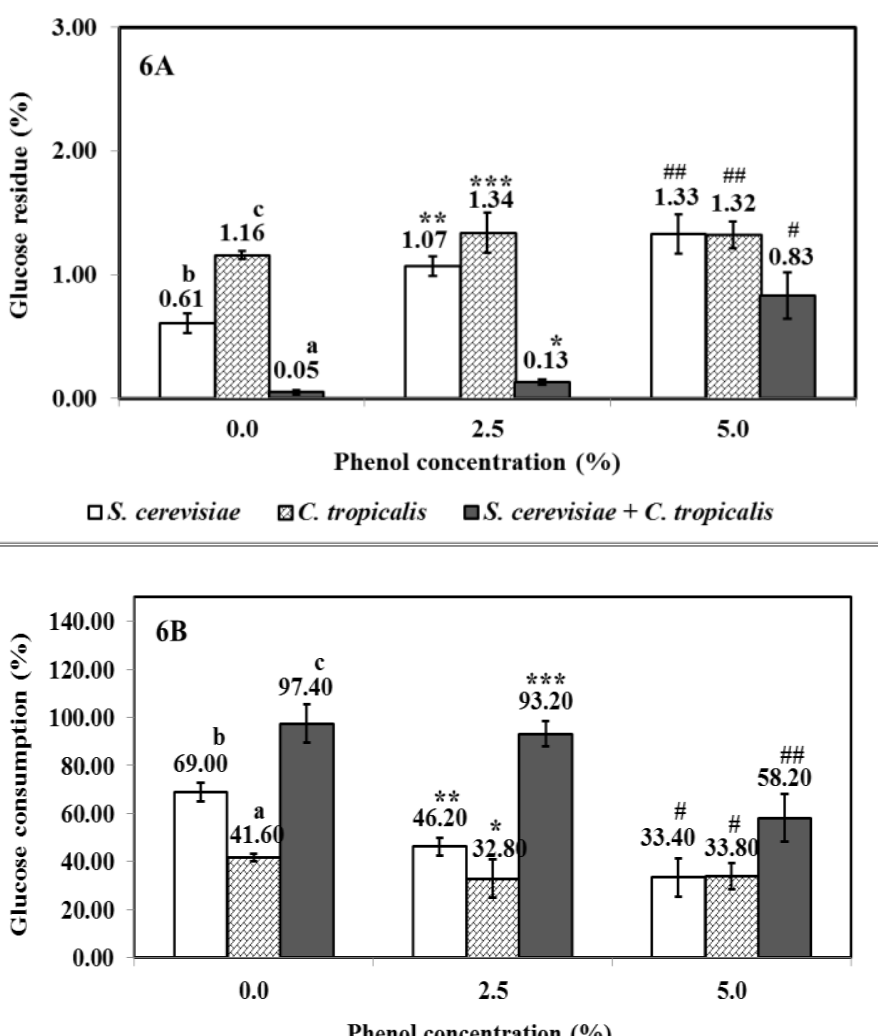

$\square$ S. cerevisiae $\quad \square$ C. tropicalis $\quad \square$ S. cerevisiae + C. tropicalis

Figure 6 Glucose residue (6A) and consumption of glucose (6B) after 5 days fermentation by mono- and co-culture of $S$. cerevisiae with $C$. tropicalis in media plus different phenol concentrations. The values with different superscripts $\left({ }^{\mathrm{a}}\right.$ and , * and ** or ${ }^{\#},{ }^{\# \#}$ and ${ }^{\# \# \#)}$ are different significant $(\mathrm{p}<0.05)$ of means from five independent observations in the same phenol concentration.
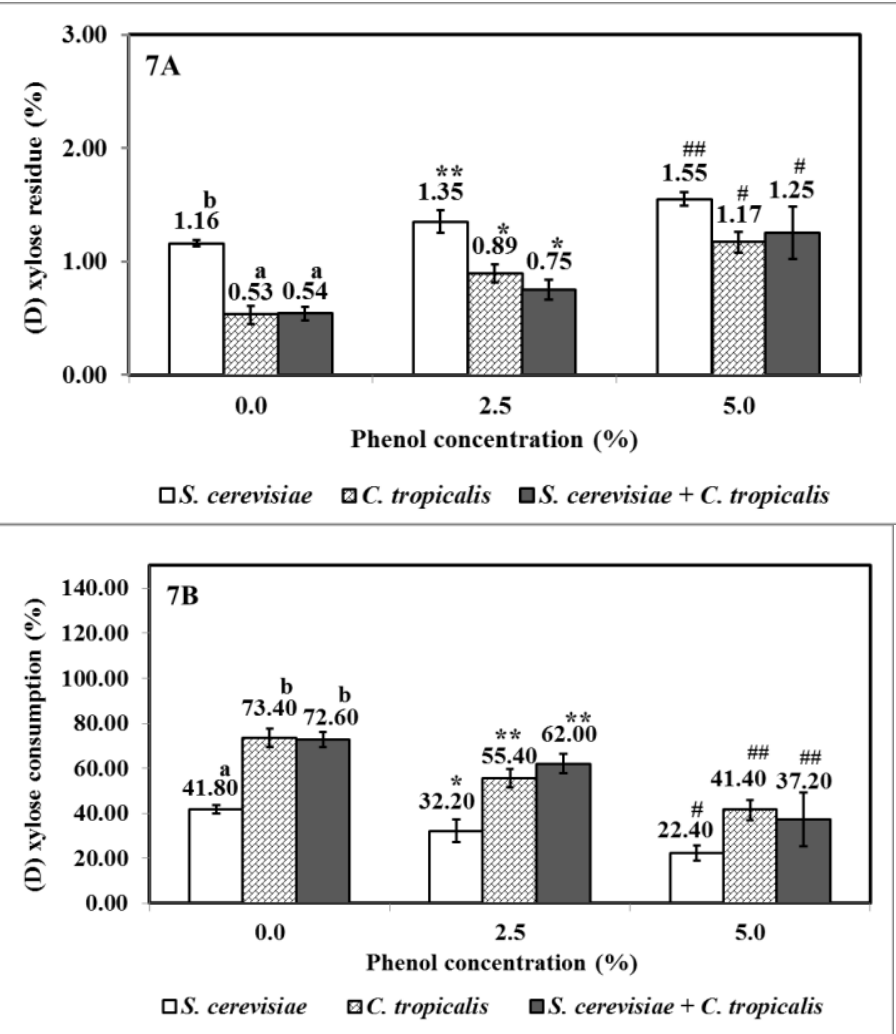

Figure 7 Xylose residue (7A) and consumption of xylose (7B) after 5 days' fermentation by mono- and co-culture of $S$. cerevisiae with $C$. tropicalis in media plus different phenol concentrations. The values with different superscripts ${ }^{\mathrm{a}}{ }^{\mathrm{a}}$ and

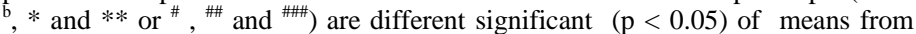
five independent observations in the same phenol concentration.
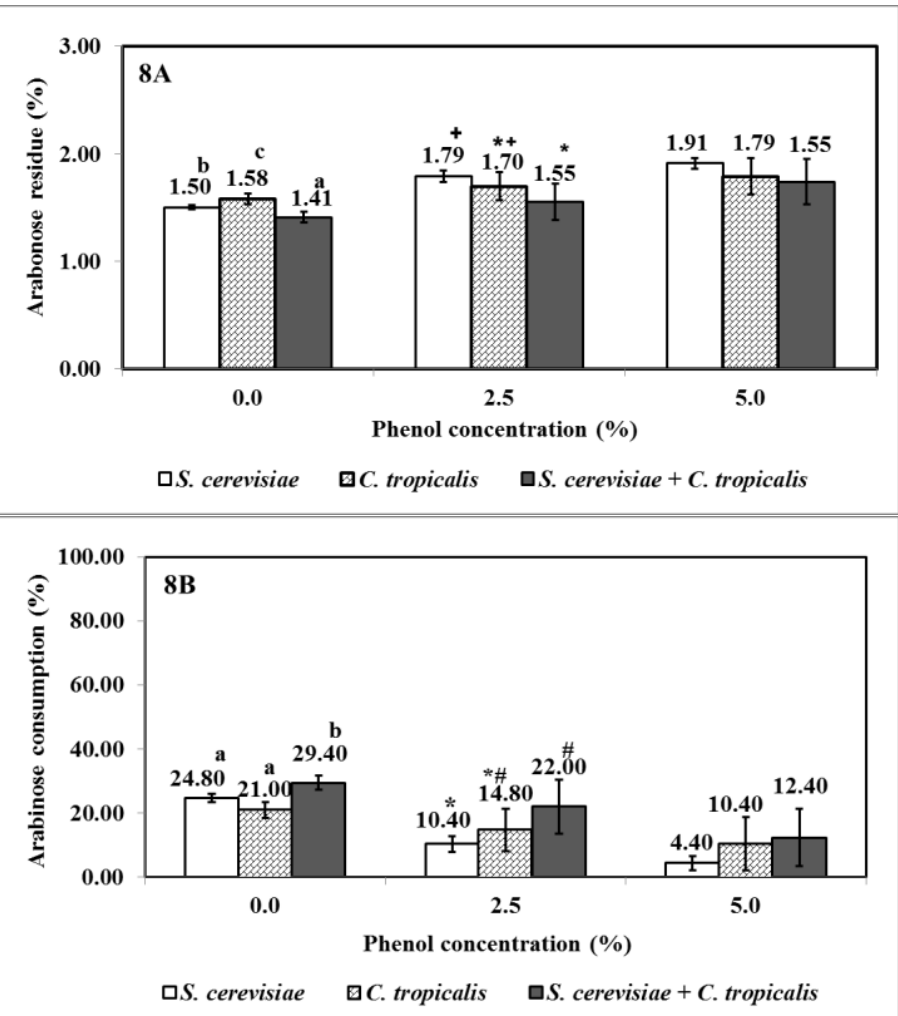

Figure 8 Arabinose residue (8A) and consumption of arabinose (8B) after 5 days' fermentation by mono- and co-culture of $S$. cerevisiae with $C$. tropicalis in media plus different phenol concentrations. The values with different superscripts $\left({ }^{\mathrm{a}}\right.$ and ${ }^{\mathrm{b}}$, or $*^{*} *^{+}$, and $\left.{ }^{+}\right)$are different significant $(\mathrm{p}<0.05)$ of means from five independent observations in the same phenol concentration.

\section{Ethanol production}

Saccharomyces cerevisiae ferments certain sugars very efficiently into ethanol, even under aerobic conditions. Addition of furfural (Fig. 9A) or phenol to the growth medium significantly $(\mathrm{p}<0.05)$ decreases ethanol yields. This work indicated that furfural inhibits sugar conversion to ethanol in mono-culture and co-culture of $S$. cerevisiea with $C$. tropicalis. Some investigators have reported an inhibitory effect of furfural on yeast growth and ethanol production. Zaldivar et al. (1999) reported that furfural and HMF compromised membrane integrity leading to extensive membrane disruption/leakage, which will eventually cause a reduction in the cell replication rate and ATP production and consequently lower ethanol production. Agbogbo and Wenger (2007) reported that a furfural concentration of $1.5 \mathrm{~g} . \mathrm{L}^{-1}$ can inhibit respiration and growth of microorganisms, leading to reduced ethanol production $(90.4 \%)$ and productivity $(85.1 \%)$. Ylitervo et al. (2013) reported that furfural in lower concentrations $(0.8$ and $1.5 \mathrm{~g}$ $\mathrm{L}-1)$ decreases ethanol yields by less than $10 \%$ and in higher concentrations decreases ethanol yield by up to around $20 \%$ and $60 \%$.

Phenolic compounds are known to partition into biological membranes, altering the permeability and lipid/protein ratio, which thus increases cell fluidity, leading to cell membrane disruption and dissipation of proton/ion gradients, thereby compromising the ability of cellular membranes to act as selective barriers (Heipieper et al., 1994). Kuntiya et al. (2013) reported that the isolate $C$. tropicalis No. 10 was fully able to degrade a phenol concentration of $100 \mathrm{mg} . \mathrm{L}^{-1}$ at $20-42{ }^{\circ} \mathrm{C}$, but this degradation was inhibited by a decreasing concentration of oxygen in media.

However, this work showed ethanol yields in the co-culture of S. cerevisiea with C. tropicalis significantly $(\mathrm{P}<0.05)$ higher than mono-culture $S$. cerevisiea or $C$. tropicalis with or without the inhibitors furfural or phenol in the medium growth We hypothesized that there is synergistic mechanism to stimulate ethanol production through simultaneous utilization of fermentable sugars such as glucose and xylose and degradation of furfural or phenol in the growth medium by co-culture $S$. cerevisiea with $C$. tropicalis. Chen (2011) suggested that coculture fermentation is a strategy for efficient conversion of glucose and xylose to ethanol and increases ethanol yield and production rate. Thurnheer et al. (1988) and Shim et al. (2002) suggested that a co-culture as a mimic of the natural environment has been used for biodegradation of aromatic compounds. Bader et al. (2010) suggested that in co-cultures, degradation and metabolization of substrates occur through the combined metabolic activity of known microbial strains under aseptic conditions. Some investigators have reported on ethanol production by co-culture of microorganisms in a medium containing furfural Wan et al. (2012) reported that co-culture of $S$. cerevisiae $\mathrm{Y} 5$ and $P$. stipitis CBS6054 effectively converted glucose and xylose to ethanol, as well as effectively degrading inhibitors in the hydrolysate. Furthermore, Wan et al. 
(2012) reported that co-culture of $S$. cerevisiae Y5 and $P$. stipitis CBS6054 used up and completely metabolized glucose, furfural and 5-HMF within $12 \mathrm{~h}$; xylose was used up in $96 \mathrm{~h}$ at $80 \mathrm{rpm}$ with ethanol concentration and yield of $27.4 \mathrm{~g}$ $\mathrm{L}^{-1}$ and $0.43 \mathrm{~g}$ ethanol $/ \mathrm{g}$ sugar without detoxification of the hydrolysate, respectively. Komarkova et al. (2003) reported that $C$. tropicalis can use a phenol concentration of $500 \mathrm{mg} \cdot \mathrm{L}^{-1}$ as a source of carbon and energy.
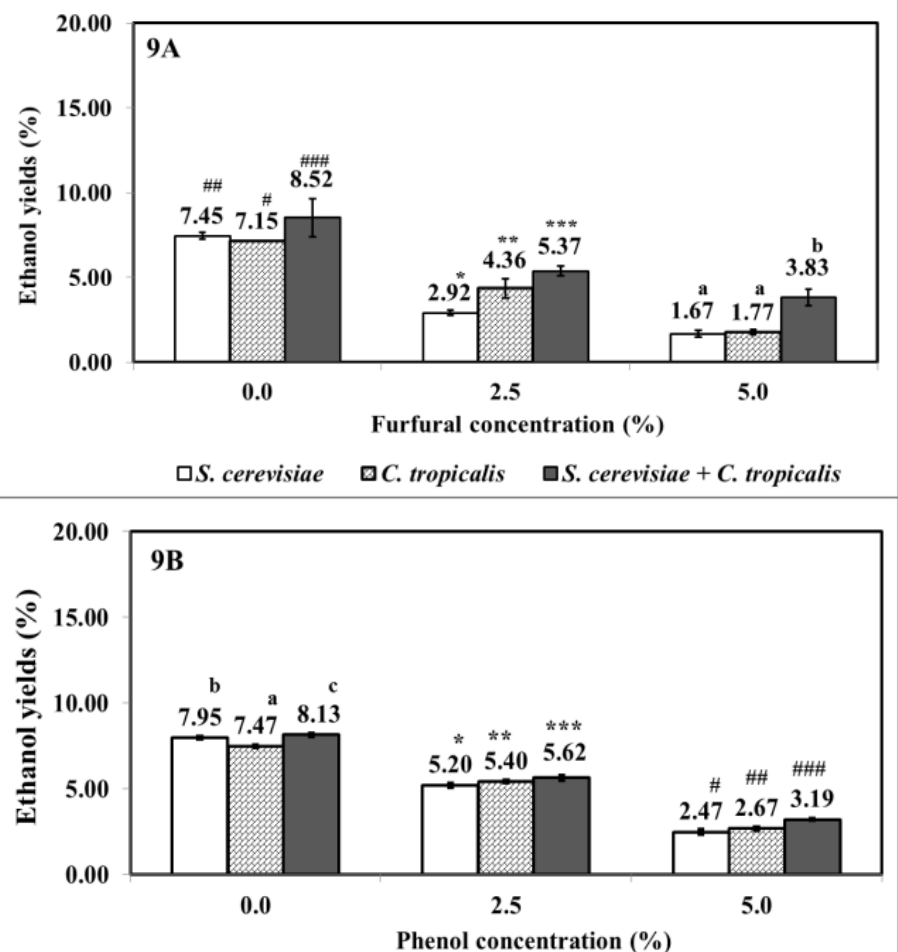

$\square$. cerevisiae $\square$ C. tropicalis $\square$ S. cerevisiae + C. tropicalis

Figure 9 Ethanol yields in media plus different furfural (9A) and phenol (9B) concentrations after 5 days' fermentation by mono- and co-culture $S$. cerevisiae with $C$. tropicalis. The values with different superscripts $\left({ }^{\mathrm{a}}\right.$ and ${ }^{\mathrm{b}}, * * *$ and $* * *$ or $\#$, \#\# and \#\#\#) are different significant $(\mathrm{p}<0.05)$ of means from five independent observations in the same furfural

\section{Efficiency of fermentation}

Addition of furfural (Fig. 10A) and phenol (Fig. 10B) to the growth medium significantly $(\mathrm{p}<0.05)$ decreased the efficiency of fermentation of ethanol production by mono- and co-culture of $S$. cerevisiae with $C$. tropicalis. However, co-culture of $S$. cerevisiae with $C$. tropicalis shown significantly $(\mathrm{p}<0.05)$ higher efficiency fermentation of ethanol production than mono-culture of $S$ cerevisiae or $C$. tropicalis from medium with or without furfural and phenol Although $S$. cerevisiae efficiently converts hexoses into ethanol, this native yeast is not able to metabolize pentose sugars present in lignocellulosic hydrolysate. This work indicates that co-culture of $S$. cerevisiae and $C$. tropicalis more efficient to use of sugar in media and convert into ethanol. Co-culture of $S$ cerevisiae with $C$. tropicalis exhibits a higher consumption of glucose and $\mathrm{D}(+)$ xylose than $C$. tropicalis and $S$. cerevisiae alone. Wang et al. (2012) suggested that co-culture between two microorganisms in a single process is an alternative way to reduce the effects of inhibitors present in the media. Hickert et al. (2012) reported that co-culture of $C$. shehatae HM 52.2 and $S$. cerevisiae ICV D254 can produce ethanol 0.42 from synthetic media and 0.51 from rice husk hydrolysate. N'Guessan et al. (2010) reported that ethanol production from sorghum by coculture of $C$. tropicalis and $S$. cerevisiae with a 2:1 ratio is higher than pure cultures of $S$. cerevisiae. Co-cultures of $S$. cerevisiae is more preferred hexose sugars consumption with yeast more preferred pentose consumption to produce ethanol efficiently is one alternative for optimizing the production of ethanol in hydrolysate containing xylose (Gutiérrez-Rivera et al., 2012; Karagoz and Ozkan, 2014; Licht, 2006). Gutiérrez-Rivera et al. (2012) reported ethanol production by co-culture of S. cerevisiae ITV-01 and Pichia stipitis NRRL Y7124 five times higher than ethanol production by mono-culture of $S$. cerevisiae ITV-01 and mono-culture of $P$. stipitis NRRL Y-7124. Increased ethanol productivity can cause enriched substrates that can be utilized as $S$. cerevisiae use glucose and $P$. stipitis use pentose to produce ethanol (Tesfaw and Assefa, 2014). Co-culture fermentation of S. cerevisiae MTCC 174 with Scheffersomyces stipitis NCIM No. 3497 can produce maximum ethanol (20.8 g. $\left.1^{-1}\right)$ higher than ethanol production by mono-culture of $S$. cerevisiae MTCC 174 (14.0 g. $\left.1^{-1}\right)$ or S. stipitis NCIM No. 3497 (12.2 g. . $^{-1}$ ) (Singh et al., 2014). Ethano production by co-culture of $S$. cerevisiae ATCC 26602 and P. stipitis DSM 3651
(7.36 g..$\left.^{-1}\right)$ shown higher than mono-culture S. cerevisiae $\left(6.68 \mathrm{~g} . \mathrm{l}^{-1}\right)$ from whea straw media with pretreatment $\mathrm{H}_{2} \mathrm{O}_{2}$ and enzyme hydrolysis (Karagoz and Ozkan, 2014). Tolerant microorganisms including co-culture fermentation to inhibitors and ethanol are one of the problems of the production of ethanol from lignocellulosic waste. Gutiérrez- Rivera et al. (2012) reported that $P$. stipitis NRRL Y-7124 has a low tolerance to ethanol produced by S. cerevisiae ITV-01 and prevents further ethanol production by $P$. stipitis NRRL Y-7124. This work showed that co-culture of $S$. cerevisiae and $C$. tropicalis has a high tolerance to inhibitors and higher ethanol yield than mono-culture of $S$. cerevisiae or monoculture of $C$. tropicalis in basal medium and basal medium plus furfural or phenol. Co-culture of $S$. cerevisiae and $C$. tropicalis also showed higher fermentation efficiency than mono-culture in basal media and basal media plus furfural or phenol. Increased ethanol production and efficiency of co-culture fermentation were allegedly due to the contribution of $C$. tropicalis to convert xylose into ethanol. Karagoz and Ozkan (2014) suggested that ethanol production was increased by co-culture of $S$. cerevisiae and $P$. stipitis due to the contribution of $P$. stipitis to convert xylose into ethanol. Hickert et al. (2013) reported that co-culture of $C$. shehatae HM 52 with $S$. cerevisiae ICV D254 in synthetic medium and rice hull hydrolysate effectively converted glucose and xylose simultaneously, maximizing the utilization rate of the substrate, and increasing the yield and rate of ethanol production.

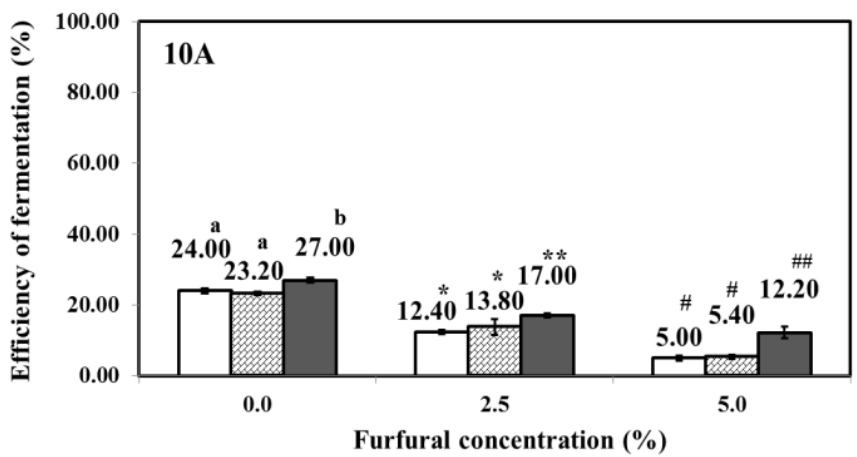

$\square$ S. cerevisiae $\quad$ C. tropicalis $\quad$ QS. cerevisiae + C. tropicalis

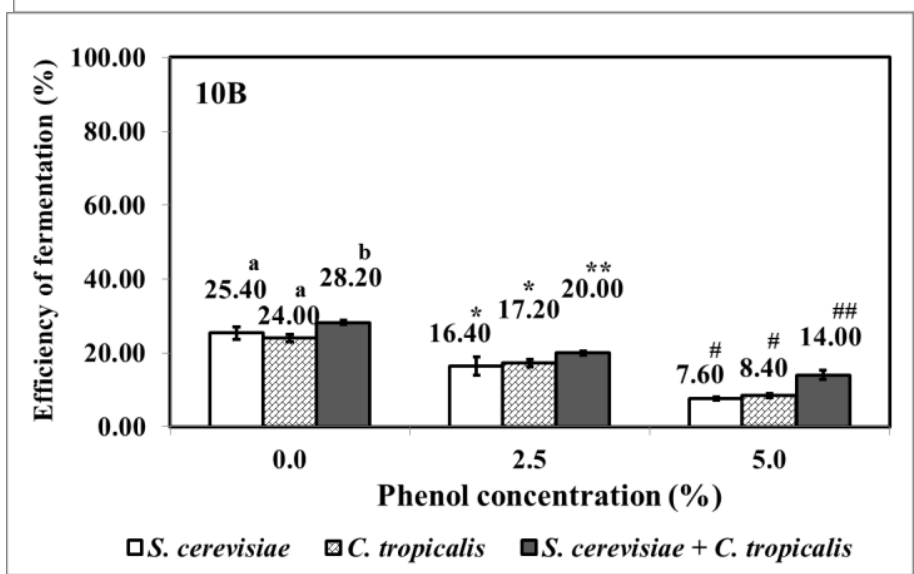

Figure 10 Efficiency of fermentation of ethanol production by mono- and coculture of $S$. cerevisiae with $C$. tropicalis from basal media plus furfural (10A) and phenol (10B) with different concentrations.

\section{CONCLUSIONS}

Sugar consumption and ethanol production by co-culture of $S$. cerevisiae with C.tropicalis in media fermentation with or without inhibitors are higher than mono $S$. cerevisiae or $C$. tropicalis. Co-culture of $S$. cerevisiae with $C$. tropicalis demonstrated a higher tolerance to inhibitor fermentation than mono-cultures for ethanol production. Glucose and xylose consumption by $S$. cerevisiae and $C$. tropicalis contribute to the improvement and efficiency of ethanol production by culture fermentation from mixed substrate. Co-culture of $S$. cerevisiae FNCC 3012 with $C$. tropicalis FNCC 3033 demonstrated potential as a fermentation process for ethanol production from lignocellulosic medium or media content inhibitors. The use of this co-culture effectively utilizes hexose and pentose sugars in the substrate, increasing the yield and efficiency of fermentation ethanol production.

Acknowledgement: The authors thank the Directorate General of Higher Education of the Ministry of Research and Higher Education, Indonesia, for funding support through its competitive research grand competition. 


\section{REFERENCES}

Abreu-Cavalheiro, A \& Monteiro, G. (2013). Solving ethanol production problems with genetically modified yeast strains. Braz. J. of Microbiol, 44 (3) 665-671. http://dx.doi.org/10.1590/S1517-83822013000300001.

Adeboye, P.T., Bettiga, M, \& Olsson, L. (2014). The chemical nature of phenolic compounds determines their toxicity and induces distinct physiological responses in Saccharomyces cerevisiae in lignocellulose hydrolysates. $\underline{A M B}$ Express. 4(46):1-10. http://dx.doi.org/10.1186/s13568-014-0046-7

Agbogbo, F.K \& Wenger, K.S. (2007). Production of ethanol from corn stover hemicellulose hydrolyzate using Pichia stipitis. J. Indus. Microbiol and Biotechnol, 34(11), 723-727.

Ahuatzi-Chacon, D., Ordorica-Morales, G., Ruiz-Ordaz, N., Cristiani-Urbina, E., Juarez-Ramirez, C., Galindez-Mayer, J. (2004). Kinetic study of phenol hydroxylase and catechol 1,2-dioxygenase biosynthesis by Candida tropicalis cells grown on different phenolic substrates. World J Microbiol Biotechnol 20(7):695-702. http://link.springer.com/article/10.1007/s11274-004-2622-5.

Almeida, J.R.M., Modig, T., Petersson,A., Hähn-Hägerdal, B., Lidén, G \& Gorwa-Grauslund, M.F.(2007). Increased tolerance and conversion of inhibitors in lignocellulosic hydrolysates by Saccharomyces cerevisiae. Mini-Review. Chem Technol and Biotechnol, 82(4): 340-349. http://dx.doi.org/10.1002/jctb.1676.

Almeida, J.R.M., Bertilsson, M., Gorwa-Grauslund, M.F., Gorsich, S \& Lidén, G. (2009). Metabolic effects of furaldehydes and impacts on biotechnologica processes. Appl Microbiol Biotechnol,82:625-638. http://dx.doi.org/10.1007/s00253-009-1875-1.

Ando, S., Arai, I., Kiyoto, K. \& Hanai, S. (1986). Identification of aromatic monomers in steam-exploded poplar and their influences on ethanol fermentation bySaccharomyces cerevisiae. J. Ferment. Technol. 64, 567-570. http://dx.doi.org/10.1016/0385-6380(86)90084-1

Bader, J., Mast-Gerlach, E., Popović, M.K., Bajpai, R. \& Stahl, U. (2010) Relevance of microbial coculture fermentations in biotechnology. Review Article. J. Applied Microbiol. 109:371-387. http://dx.doi.org/10.1111/j.13652672.2009.04659.

Balat, M. (2011). Production of bioethanol from lignocellulosic materials via the biochemical pathway: A review. Energy Conversion and Management 52(2):858-875.

Bettiga, M., Bengtsson, O., Hahn-Hägerdal, B \& Gorwa-Grauslund, M.F. (2009). Arabinose and xylose fermentation by recombinant Saccharomyces cerevisiae expressing a fungal pentose utilization pathway. Microbial Cell Factories, 8:40 http://dx.doi.org/10.1186/1475-2859-8-40.

Cardona, C.A. \& Sa'nchez, O.J. (2007). Fuel ethanol production: process design trends and integration opportunities. Bioresour. Technol. 98:2415-2457. http://dx.doi.org/10.1016/j.biortech.2007.01.002

Caspeta, L., Castillo, T. \& Nielsen, J. (2015). Modifying Yeast Tolerance to Inhibitory Conditions of Ethanol Production Processes. Review. Front. Bioeng. Biotechnol., 3:1-15. http://dx.doi.org/10.3389/fbioe.2015.00184

Chen, Y. (2011). Development and application of co-culture for ethanol production by co-fermentation of glucose and xylose: a systematic review. $\underline{J}$ Ind Microbiol Biotechnol, 38(5):581-97. http://dx.doi.org/10.1007/s10295-010-0894-

Cheng, K.K., Wu, J., Zhang-Nan, L \& Zhang, Jian-An. (2014). Aerobic and sequential anaerobic fermentation to produce xylitol and ethanol using nondetoxified acid pretreated corncob. Biotechnology for Biofuels. 7:166.

Chiang, L.C., Gong, C.S., Chen, L.F. \& Tsao, G.T. (1981). D-xylulose fermentation to ethanol by Saccharomyces cerevisiae. Appl Environ Microbiol. 42:284-289.

Delgenes, J. P., Moletta, R., and Navarro, J. M. (1996). Effects of lignocellulose degradation products on ethanol fermentations of glucose and xylose bySaccharomyces cerevisiae, Zymomonas mobilis, Pichia stipitis, and Candida shehatae. Enzyme Microb. Technol. 19, 220-225. http://dx.doi.org/10.1016/0141 0229(95)00237-5

Ding, M.Z., Wang, X., Yang, Y \& Yuan, Y.J. (2011). Metabolomic study of interactive effects of phenol, furfural, and acetic acid on Saccharomyces cerevisiae. OMICS, 15(10):647-53. http://dx.doi.org/10.1089/omi.2011.0003.

Diaz De Villegas, M.E, Villa, P., Guerra, M., Rodríguez, E., Redondo, D \& Martinez, A. (1992). Conversion of furfural into furfuryl alcohol by Saccharomyces cerevisiae.Acta Biotechnol, 12:351-354. http://dx.doi.org/ 10.1002/abio.370120420.

Du Preez, J.C., Bosch, M \& Prior, B.A. (1986). Xylose fermentation by C shehatae and Pichia stipites: effects of $\mathrm{pH}$, temperature and substrate concentration. Enzyme Microb Technol, 8:360-364.

Eiteman, M.A., Sarah, A.L \& Altman, E. (2008). A co-fermentation strategy to consume sugar mixtures effectively. J Biol Eng, 2(3): 1-8 http://dx.doi.org/10.1186/1754-1611-2-3

Fu, N. \& Peiris, P. 2008. Co-fermentation of a mixture of glucose and xylose to ethanol by Zymomonas mobilis and Pachysolen tannophilus. World J Microbio and Biotechnol. 24(7):1091-1097. link.springer.com/article/10.1007/s11274-0079613-2
Gong, C.S., Chen, L.F., Flickinger, M.C., Chiang, L.C. \& Tsao, G.T. (1981) Production of ethanol from D-xylose by using D-xylose isomerase and yeasts. Appl Environ Microbiol. 41:430-436.

Gutiérrez-Rivera, B., Waliszewski-Kubiak, K., Carvajal-Zarrabal, O \& AguilarUscanga, M.G. (2012). Conversion efficiency of glucose/xylose mixtures for ethanol production using Saccharomyces cerevisiae ITV01 and Pichia stipitis NRRL Y-7124. J. Chem Technol and Biotechnol. 87(2): 263-270. http://dx.doi.org/10.1002/jctb.2709.

Heipieper, H.J., Weber, F.J., Sikkema, J., Kewelo, H \& de Bont. J.A.M. (1994) Mechanism of resistance of whole cells to toxic organic solvents. Trends Biotech 12:409-415

Hickert, L.R., Da Cunha-Pereira, F., De Souza-Cruz, P. B., Rosa, C. A. \& Ayub. M. A. Z. (2013). Ethanogenic fermentation of co-cultures of Candida shehatae HM 52.2 and Saccharomyces cerevisiae ICV D254 in synthetic medium and rice hull hydrolysate. Bioresource Technol. 131: 508 514. http://dx.doi.org/10.1016/j.biortech.2012.12.135.

Hristozova, Ts., Gotcheva, V., Tzvetkova, B., Paskaleva, D. \& Angelov, A (2000). Effect of furfural on nitrogen assimilating enzymes of the lactose utilizing yeasts Candida blankii 35 and Candida pseudotropicalis 11. $\underline{\text { J Biosci }}$ Bioeng. 90(4):374-80. http://dx.doi.org/10.1016/j.enzmictec.2008.03.014.

Ishola, M.M., Isroi \& Taherzadeh, M.J. (2014) Effect of fungal and phosphoric acid pretreatment on ethanol production from oil palm empty fruit bunches (OPEFB) Technol.

165:9-12.

http://dx.doi.org/10.1016/j.biortech.2014.02.053.

Jeffries, T.W \& Jin, Y.S. (2004). Metabolic engineering for improved fermentation of pentoses by yeasts. Appl Microbiol Biotechnol 63:495-509. http://dx.doi.org/10.1007/s00253-003-1450-0.

Jones, R.P (1989). Biological principles for the effects of ethanol. Enzyme Microb Technol. 11:130-153.

Jönsson, L.J., Alriksson, B \& Nilvebrant, N.O. (2013). Bioconversion of lignocellulose: inhibitors and detoxification, Review. Biotechnology for Biofuels. 6:16, 1-10. http://dx.doi.org/10.1186/1754-6834-6-16.

Karagoz, K \& Ozkan, M. (2014). Ethanol production from wheat straw by Saccharomyces cerevisiae and Scheffersomyces stipitis co-culture in batch and continuous system. Bioresource Technol. 158: 286-293. http://dx.doi.org/10.1016/j.biortech.2014.02.022.

Kelly, C., Jones, O., Barnhart, C \& Lajoie, C. (2008). Effect of furfural, vanillin and syringaldehyde on Candida guilliermondii growth and xylitol biosynthesis Appl Biochem Biotechnol. 148(1-3):97-108. http://dx.doi.org/10.1007/s12010 007-8103-1.

Keweloh, H., Weyrauch, G., and Rehm, H.-J. (1990). Phenol-induced membrane changes in free and immobilized Escherichia coli.Appl. Microbiol. Biotechnol. 33, 66-71. http://dx.doi.org/10.1007/bf00170572

Klinke, H. B., Thomsen, A. B., and Ahring, B. K. (2004). Inhibition of ethanolproducing yeast and bacteria by degradation products produced during pretreatment of biomass. Appl. Microbiol. Biotechnol. 66, 10-26 http://dx.doi.org/10.1007/s00253-004-1642-2

Komarkova, E., Paca, J., Klapkova, E., Stiborova, M., Soccol, C.R \& Sobotka, M. (2003). Physiological changes of Candida tropicalis population degrading phenol in fed batch reactor. Braz. arch. biol. Technol, 46(4):537-543. http://dx.doi.org/10.1590/S1516-89132003000400007.

Krug, M., Ziegler, H, \& Straube, G. (1985). Degradation of phenolic compounds by yeast Candida tropicalis HP15. I. Physiology of growth and substrate utilization. $J$ Basic Microbiol.

25:103-10 http://dx.doi.org/10.1002/jobm.3620250206

Kumar, R., Singh, S \& Singh, O.V. (2008). Bioconversion of lignocellulosic biomass: biochemical and molecular perspectives. J Ind Microbiol Biotechnol, 35:377-391.

Kuntiya, A., Takenaka, S \& Seesuriyachan, P. (2013). High potential of thermotolerant Candida tropicalis no. 10 for high concentration of phenol biodegradation. Food and Appl Bios J, 1(2): 59-68.

Laplace, J.M., Delgenes, J.P., Moletta, R. \& Navarro, J.M. (1992). Alcoholic glucose and xylose fermentation by the coculture process: compatibility and typing of associated strains. Can $J$ Microbiol. 38:654-658. http://dx.doi.org/10.1139/m92-106

Laplace, J. M., Delgenes, J. P.,Moletta, R., \& Navarro, J.M. (1993) Cofermentation of glucose and xylose to ethanol by a respiratory-deficien mutant of Saccharomyces cerevisiae co-cultivated with a xylose-fermenting yeast, J. Ferment. Bioeng. 75; 207-212.

Larsson, S., Palmqvist, E., Hahn-Hägerdal, B., Tengborg, C., Stenberg, K. Zacchi, G \& Nilvebrant, N-O. (1999). The generation of fermentation inhibitors during dilutes acid hydrolysis of softwood. Enzyme Microb Technol. 24:151159. http://dx.doi.org/10.1016/S0141-0229(98)00101-X.

Larsson, S., Cassland P \& Jonsson, L.J. (2001). Development of a Saccharomyces cerevisiae strain with enhanced resistance to phenolic fermentation inhibitors in lignocellulose hydrolysates by heterologous expression of laccase. Appl and Environ. Microbiol, 67(3): 1163-1170.

Larsson, S., Quintana-Sáinz A, Reimann A, Nilvebrant N-O, \& Jönsson LJ. (2000). Influence of lignocellulose-derived aromatic compounds on oxygen 
limited growth and ethanolic fermentation by Saccharomyces cerevisiae. Appl Biochem Biotechnol. 84:617-632.

Lee, W.C \& Huang, C.T. (2000). Modeling of ethanol fermentation using Zymomonas mobilis ATCC 10988 grown on the media containing glucose and fructose. Biochem Eng J, 4:217-227.

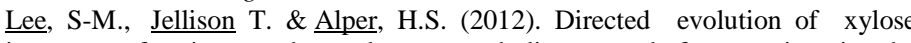
isomerase for improved xylose catabolism and fermentation in the yeast Saccharomyces cerevisiae. Appl Environ Microbiol, 78(16): 5708-5716. http://dx.doi.org/10.1128/AEM.01419-12.

Licht, F. (2006). World Ethanol Markets: The Outlook to 2015, Agra Europe, Tunbridge Wells, UK.

Lin, Y \&. Tanaka, S. (2006). Ethanol fermentation from biomass resources: current state and prospects. Appl Environ Microbiol, 69:627-642.

Lin, R., Cheng, J., Ding, L., Song, W., Zhou, J, \& Cen, K. (2015). Inhibitory effects of furan derivatives and phenolic compounds on dark hydrogen fermentation. http://dx.doi.org/10.1016/j.biortech.2015.07.097.

Mattam, A.J., Kuila, A., Suralikerimath, N., Choudary, N., Peddy, V., Rao, C \& Velankar, H.R. (2016). Cellulolytic enzyme expression and simultaneous conversion of lignocellulosic sugars into ethanol and xylitol by a new Candida tropicalis strain. Biotechnol Biofuels. 2016; 9: 157. doi: 10.1186/s13068-0160575-1.

Mielenz, J.R. (2001). Ethanol production from biomass: technology and commercialization status. Curr Opin Microbiol, 4:324-329. http://dx.doi.org/10.1016/S1369-5274(00)00211-3.

Mills, C., Child, J. J., and Spencer, J. F. T. (1971). The utilization of aromatic compounds by yeasts. Antonie Van Leeuwenhoek 37, 281-287. http://dx.doi.org/10.1007/bf02218497

N'Guessan, F.K., N'Dri, D.Y., Camara, F.\& Djè, M. K. (2010). Saccharomyce cerevisiae and Candida tropicalis as starter cultures for the alcoholic fermentation of tchapalo, a traditional sorghum beer. World J Microbiol and Biotechnol. 26(4):693-699.

Páca, J., Komárková, E., Prell, A., Stiborová, M \& Sobotka, M. (2002). Kinetics of phenol oxidation by Candida tropicalis: Effects of oxygen supply rate and nutrients on phenol inhibition. Folia Microbiologica. 47(6): 701-707.

Panchal, C.J., Bast, L., Russell, I, \& Stewart, G.G. (1988). Repression of xylose utilization by glucose in xylose-fermenting yeasts. Can J Microbiol, 34:1316 1320. http://dx.doi.org/10.1139/m88-230.

Palmqvist, E., \& Hahn-Hägerdal, B. (2000). Fermentation of lignocellulosic hydrolysates II: inhibitors and mechanisms of inhibition. Bioresour Technol, 74:25-33. http://dx.doi.org/10.1016/S0960-8524(99)00161-3.

Parawira, W \& Tekere, M. (2011). Biotechnological strategies to overcome inhibitors in lignocellulose hydrolysates for ethanol production: review. Crit Rev Biotechnol. 1(1):20-31. http://dx.doi.org/10.3109/07388551003757816.

Pizzolitto, R.P., Barberis, C.L., Dambolena, J.S., Herrera, J.M., Zunino, M.P., Magnoli, C.E., Rubinstein, H.R., Zygadlo, J.A, \& Dalcero, A. M. (2015) Inhibitory effect of natural phenolic compounds on Aspergillus parasiticus growth. Journal of Chemistry 2015:1-7. https://www.hindawi.com/journals/jchem/2015/547925/.

Richard, P., Verho, R., Putkonen, M., Londesborough, J. \& Penttilä. (2003). Production of ethanol from L-arabinose by Saccharomyces cerevisiae containing a fungal L-arabinose pathway. FEMS yeast research.3(2):185-189. http://dx.doi.org/10.1016/S1567-1356(02)00184-8

Sánchez, S., Bravo, V., Castro, E., Moya, A.J. \& Camacho. F. (2002). The fermentation of mixtures of D-glucose and D-xylose by Candida shehatae, Pichia stipites or Pachysolen tannophilus to produce ethanol. J. Chem Techno Biotechnol. 77:641-648. http://dx.doi.org/10.1002/jctb.622.

Sárvári Horváth, I., Franzén, C.J., Taherzadeh, M.J., Niklasson, C, \& Lidén, G. (2003). Effects of furfural on the respiratory metabolism of Saccharomyce cerevisiae in glucose-limited chemostats. Appl Environ Microbiol,69:4076-4086. Schimer-Michel, A.C., Flôres, S.H., Hertz, P.F., Matos, G.S, \& Ayub, M.A.Z (2008). Production of ethanol from soybean hull hydrolysate by osmotolerant Candida guilliermondii NRRL Y-2075. Bioresour. Technol., 99: 2898-2904. http://dx.doi.org/10.1016/j.biortech.2007.06.042

Service, R.F. (2007). Cellulosic ethanol: biofuel researchers prepare to reap a new harvest. Science 315:1488-1491.

Shim, H., Shin, E.B.\& Yang, S.T. (2002). A continuous fibrous-bed bioreactor for BTEX biodegradation by a co-culture of Pseudomonas putida and Pseudomonas fluorescens. Adv Environ Res. 7:203-216.

Shin, H.Y., Nijland, J.G, de Waal, P.P, de Jong, R.M., Klaassen, P. \& M. Driessen, A.J.M. (2015). An engineered cryptic Hx11 sugar transporter facilitates glucose-xylose co-consumption in Saccharomyces cerevisiae. Biotechnology for Biofuels. 8:176. http://dx.doi.org/10.1186/s13068-015-0360-6. Singh, A., Bajar, S \& Bishnoi, N.R.. (2014). Enzymatic hydrolysis of microwave alkali pretreated rice husk for ethanol production by Saccharomyces cerevisiae, Scheffersomyces stipitis and their co-culture. Fuel, Vol. 116: 699-702. Sluiter, J.B., Ruiz, R.O., Scarlata, C.J., Sluiter, A.D \& Templeton. D.W.(2010) Compositional analysis of lignocellulosic feedstocks. 1. Review and description of methods. J Agric Food Chem. 58:90439053. http://dx.doi.org/10.1021/if1008023.
Sopandi, T \& Wardah, A. (2015). Sugar consumption in mono and co-culture Saccharomyces cerevisiae and others selected microorganism for bioethano production from stream rice husk medium. Asian Jr. of Microbiol. Biotech. Env. Sc. $17(3): 2015: 89-98$

Taherzadeh, M.J., Gustafsson, L., Niklasson, C, \& Lidén, G. 1999. Conversion of furfural in aerobic and anaerobic batch fermentation of glucose by Saccharomyces cerevisiae. J. Biosci and Bioeng. 87(2): 169-174. http://dx.doi.org/10.1016/S1389-1723(99)89007-0.

Taherzadeh, M.J., Gustafsson, L., Niklasson, C \& Lidén, G. (2013). Inhibition effects of furfural on aerobic batch cultivation of Saccharomyces cerevisiae growing on ethanol and/or acetic acid. Bioresour Technol. 131:508-14 http://dx.doi.org/10.1016/j.biortech.2012.12.135.

Taniguchi, M., Tohma, T., Itaya,T \& Fujii, M. (1997). Ethanol production from a mixture of glucose and xylose by co-culture of Pichia stipites and a respiratory deficient mutant of Saccharomyces cerevisiae. J Ferment Bioeng. 83:364-370. http://dx.doi.org/10.1016/S0922-338X(97)80143-2

Tesfaw, A \& Assefa, F.(2014). Current trends in bioethanol production by Saccharomyces cerevisiae: substrate, inhibitor reduction, growth variables, coculture, and immobilization. Int. Scho. Res. Not. 2014:1-11. https://www.hindawi.com/journals/isrn/2014/532852/

Thurnheer, T., Cook, A.M., Leisinger, T. (1988). Co-culture of defined bacteria to degrade seven sulfonated aromatic compounds: efficiency, rates and phenotypic variations. Appl Microbiol Biotechnol, 29:605-609.

Tomás, A.F., Karagöz, P., Karakashev, D, \& Angelidaki, I. (2013). Extreme thermophilic ethanol production from rapeseed straw: using the newly isolated Thermoanaerobacter pentosaceus and combining it with Saccharomyces cerevisiae in a two-step process. Biotechnol and Bioeng:110 (6): 1574-1582. http://dx.doi.org/10.1002/bit.24813

van Maris, A.J.A., Abbott, D.A., Bellissimi, E., van den Brink. J., Kuyper, M., Luttik, M.A.H., Wisselink, H.W., Scheffers, W.A., van Dijken, J.P. \& Pronk, J.T.(2006). Alcoholic fermentation of carbon sources in biomass hydrolysates by Saccharomyces cerevisiae: current status. Antonie Van Leeuwenhoek Int J Gen Mol Microbiol, 90:391-418.

Wan. P., Zhai. D, Wang, Z., Yang, X. \& Tian, S. (2012). Ethanol production from nondetoxified dilute-Acid lignocellulosic hydrolysate by cocultures of Saccharomyces cerevisiae Y5 and Pichia stipitis CBS6054. Biotechnol Res. Inter. 2012:6-11. http://dx.doi.org/10.1155/2012/656371.

Wang, J., Mab, X., Liub, S., Sunc, P., Fanc, P, \& Xiab, C. (2012). Biodegradation of phenol and 4-chlorophenol by Candida tropicalis W1. The 7th Inter Conf. on Waste Manag and Technol. Proc Environ Sci. 16: 299 - 303. http://dx.doi.org/10.1016/j.proenv.2012.10.042.

Wahlbom CF, Hahn-Hägerdal B. (2002). Furfural, 5-hydroxymethyl furfural, and acetoin act as external electron acceptors during anaerobic fermentation of xylose in recombinant Saccharomyces cerevisiae. Biotech Bioeng. 78(2):172178. http://dx.doi.org/10.1002/bit.10188

Wendland, J., Schaub, Y, \& Walther, A. (2009). N-Acetylglucosamine Utilization by Saccharomyces cerevisiae Based on Expression of Candida albican $N A G$ Genes. Appl and Environ Microbiol, 75(18): 5840-5845.

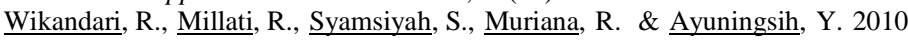
Effect of Furfural, Hydroxymethylfurfural and Acetic Acid on Indigeneous Microbial Isolate for Bioethanol Production. Agric.Journal. 5(2): 105-109. http://www.medwelljournals.com/fulltext/?doi=aj.2010.105.109

Ylitervo, P., Franzén, C.J, \& Taherzadeh, M.J. (2013). Impact of Furfural on Rapid Ethanol Production Using a

$\begin{array}{llll}\text { Membrane } & \text { Bioreactor. } & \text { Energies. }\end{array}$ http://www.dx.doi.org/10.3390/en6031604

Zaldivar, J., Martinez, A. \& Ingram, L.O. (1999). Effect of selected aldehyde on the growth and fermentation of ethanologenic Escherichia coli LY01. Biotechnol Bioeng. 65:24-33

Zaldivar, J., Nielsen, J., \& Olsson, L. (2001). Fuel ethanol production from lignocellulose: a challenge for metabolic engineering and process integration. Appl Microbiol Biotechnol, 56:17-34. 